\title{
Offline Handwritten Hindi Word Recognition
}

\author{
${ }^{1}$ Nistha Lodhi, ${ }^{2}$ Vikas Singhal \\ Department of Computer Applications \\ JSS Academy of Technical Education, Noida, India
}

\begin{abstract}
There are few approaches to character distinguishing proof. Basic stages of character recognition incorporate pre-processing, segmentation, features extraction and classification of features. A few classification techniques have been talked about in this paper, such as neural network, System Vector Machine (SVM), coordinating models, etc. The issue of character affirmation has been turned out from different viewpoints. One of the foremost well-known ways is as character grouping. Character course of action could be a zone wherein a system can recognize the assorted information boosts in an imperative gathering as shown by the highlights show within the data character. In this paper we investigate the Hindi Offline written by hand word acknowledgment (HWR) that we are making. In the HWR, we utilize OCR Framework, OCR is the strategy of taking pictures or pictures of letters or typhoid content and interpreting them into data that the computer can effectively translate and in Convolutional Neural Network (CNN). The proposed framework has been prepared on tests of a sizably voluminous set of database pictures and tried on tests pictures from utilizer characterizes information set and from this try we accomplished exceptionally tall apperception comes about.
\end{abstract}

Keyword: HWR, OCR System, feature extraction, Python, CNN.

\section{INTRODUCTION}

Offline penmanship acknowledgment includes recognizing the pictures of a word that has as of now been composed, checked, or recorded pictures employing a camera. Recognizing handwritten Hindi characters consequently could be an exceptionally troublesome errand since characters are composed in numerous ways, such as bends and cursively composed characters, which are distinctive completely different ways. OCR is the way toward taking pictures or pictures of letters or typhoid substance and changing over them into information that a machine can without a doubt translate. Manually written acknowledgment insinuates to the way toward deciphering pictures of physically composed, typhoid, or printed digits into an arrangement comprehended by the client for the reason for changing, ordering/looking, and a diminish absent measure. Physically composed affirmation system is having its importance and it is versatile in several areas, for case, webbased penmanship affirmation on PC tablets, see postal locale on mail for postal mail orchestrating, preparing, bank checksums, numeric segments in structures topped off by hand, etc.

\section{OCR SYSTEM AND CNN}

\section{a). OCR System:}

Automatic text apperception utilizing OCR is the method of changing over a picture of textual documents into its digital textual equipollent. The advantage is that the textual material may be altered, which something else isn't conceivable in scanned documents during which these are image files.

The subsequent steps are followed within the design of the proposed OCR framework:

- Pre-processing

- Segmentation

- Feature Extraction

- Classification

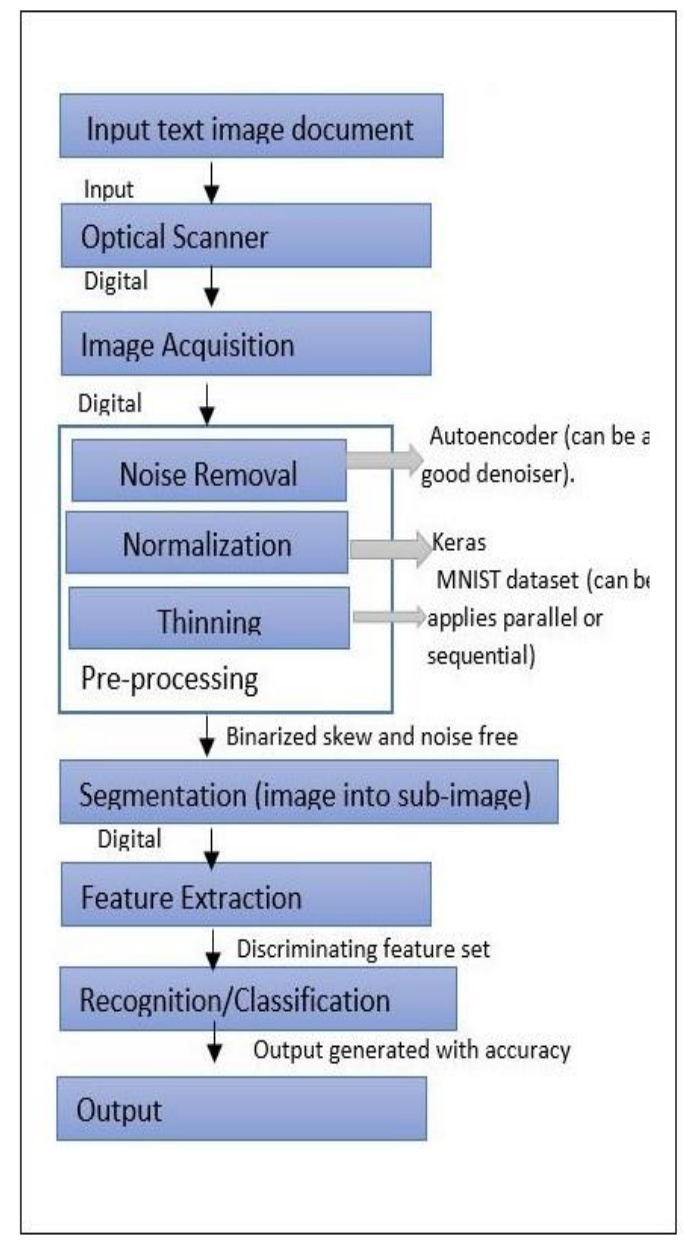

Figure 1. OCR System 


\section{Pre-processing:}

The pre-processing arrange produces a straightforward archive inside the sense that the foremost shape data with negligible clamor and greatest compression on the normalized picture is gotten.

After securing the picture, it'll be handled through the grouping of handling steps:

- Noise Removal: Reducing noise from image. In online there's no noise, hence there's must not eliminate noise. But in offline the noise could also become from sort or from the gadget which captures the image.

- Normalization: Standardize the textual style measures inside the picture. This issue shows up clearly inside the written by hand content since the textual style measure isn't confined when utilizing handwriting.

- Thinning: Thinning algorithm will be parallel or consecutive. The parallel is connected to all the pixels simultaneously.

Consecutive look at pixels and changing them wagering on the going before handled result.

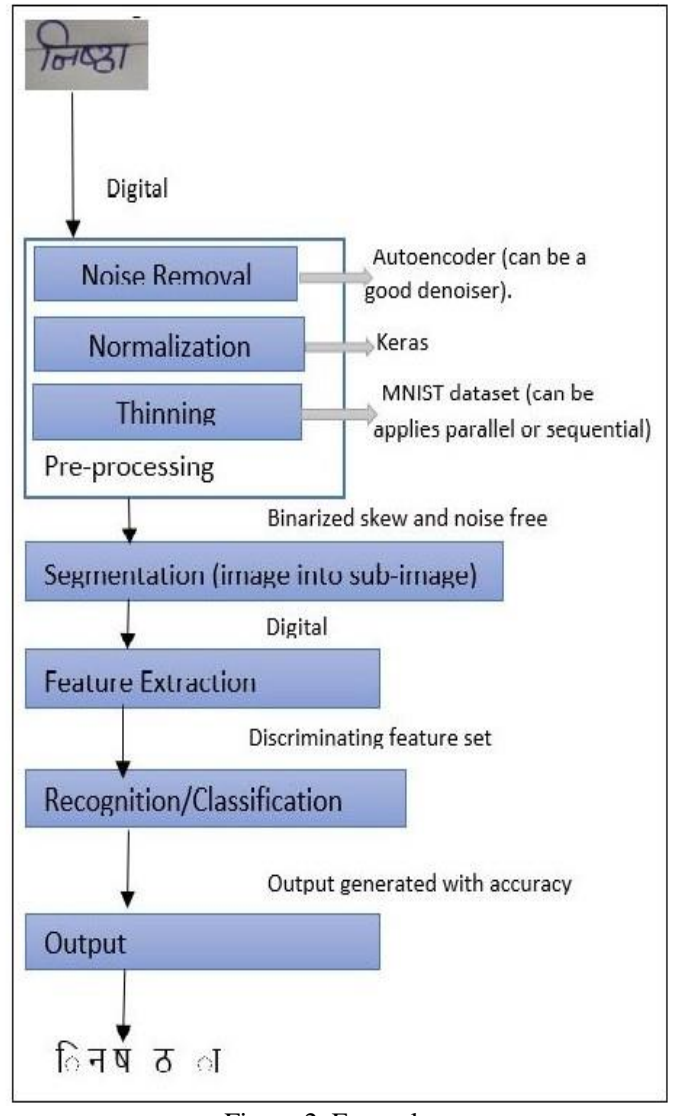

Figure 2. Example

\section{Segmentation:}

Inside the segmentation organize, a picture comprising of a grouping of characters is reduced into sub-images of a tactful character. This stage comprises of fragmenting the preprocessed and clean record into its sub-components. Segmentation may be exceptionally vital stage, since of the acknowledgment of manually characters straightforwardly influenced by how the partition of lines into words, words into letters/characters can be viably achieved.

\section{Feature Extraction:}

Extraction of features from the sectioned individual characters may be a very vital step of the acknowledgment framework since highlight sets play one of the foremost vital parts in an acknowledgment framework. A great highlight set speaks to specifically the characteristics of a course which makes a difference to segregate against other classes whereas the characteristics of lesson components inside a lesson stay the same. The fundamental purpose of this phase is to choose the most excellent include set, which minimizes the blunder of acknowledgment with an ideal set of features.

Classification: The back-propagation algorithm is employed for classification or identification. The classification is apportioned on the thought of the extricated highlights. For the inceptive classification of characters, considered three highlights are as follows:

- Projection histogram supported pixel value

- Distance

- Supported the spatial position of the pixel Projection histogram.[2].

\section{b). CNN Architecture:}

CNN may be a bunch of three fundamental layers which are: convolution layer, pooling layer or sub-sampling layer and classification layer or thick layer.

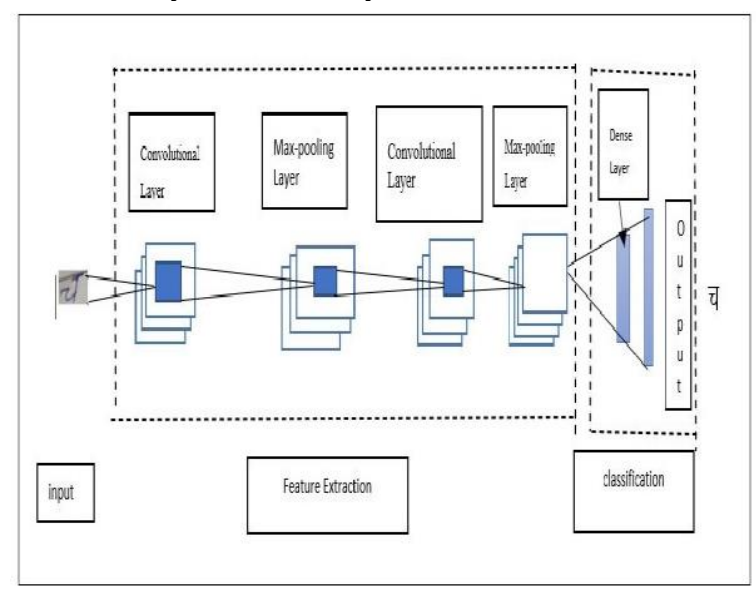

Figure 3. CNN Architecture

The to begin with step required to start the distinguishing proof is to separate a hand indited character picture for assignment. The input layer will carry the crude pixel values of the winnowed picture of tallness and width $32 \times 32$ for Hindi. At that point it passes the input picture to the convolution layer. The obligation of this layer is to include a subjective number of channels to continue along the stature and width of the picture to abdicate an included outline. (A channel will be an arrangement of numbers called parameters or weights). An audit weights learned from the distinctive layers of the proposed expanded picture platform. 
A work is gotten by sliding through the channel through the channel through the picture tallness and separate, and the dab estimation item between the volume of the input and the channel amid the front pass.

We have accomplished a character 32X32 in Hindi. The yield of the primary convolutional layer incites 4 highlighted maps in Hindi and changes it to another layer through a differentiable work. Finally, the yield is of 3D $32 \times 32 X 4$ which is changed to be begin with pooling layer where the picture is down sampled along the spatial measurements coming about in a yield volume of 28X28X4 for Hindi [3].

\section{BACKGROUND}

Nisha Vasudeva et al. [2012] The development of AI has driven to the development of numerous "shrewd" gadgets. The foremost imperative challenge inside the field of picture handling is to recognize archives in both written by hand and printed groups. Character acknowledgment is one of the biometric highlights frequently utilized for the confirmation of both a person and an archive. OCR a sort of report picture examination was inspected with a modern picture containing either machine-printed or translated substance as a commitment to the OCR programming engine and changed over into a balanced machine-comprehensible computerized substance course of action. For the calculation of the include, we connected a include extraction procedure. Extricated character highlights are pixel rules for his or her neighboring pixels. And these inputs give a neural back spreading organize with a covered up and yield layer. Back spread neural arrange for redress acknowledgment where mistakes are made strides.

Sonal P Ajmire et al. [2015] the concept of character recognition has gotten an amazing bargain of consideration much obliged to its numerous applications, like filling out different shapes, in printed postal addresses, multiple-choice questions in a few examinations, and so on. This paper may be a well-defined consider of the written by hand acknowledgment of Devanagari characters. It characterizes organized strategies for the ubiquity of written by hand characters for the extraction of highlights. Character acknowledgement may too be of two sorts that are offline and online character acknowledgment. This will indeed be classified as transcribed and printed character acknowledgement. Compared to the notoriety of printed characters. There are more applications for manually written character acknowledgment.

\section{MNIST Dataset:}

\section{METHODOLOGY}

This dataset was made by blending distinctives sets interior the introductory National Established of measures and Innovation (NIST) sets, so on have a preparing set containing a few sorts and shapes of manually written digits, since the NIST set was separated into those composed by high school understudies et.al. composed by the office workers. Indeed, TensorFlow and Keras authorize us to purport and download the MNIST dataset straightforwardly from their API. Consequently, I'll commence with the taking after two lines to purport TensorFlow and MNIST dataset beneath the Keras API

\section{PYTHON:}

Python is broadly utilized for information examination, but the information does not continuously need to be within the organize required. In these occasions, we change over the text document to the filtered report for a clearer investigation of the comes about. Python incorporates a part of libraries to do this (libraries like Keras, Pandas, Numpy).

Libraries that we used:

- Keras.

- TensorFlow

\subsection{Keras:}

Keras is a high-level neural networks API planned with a center on permitting quick experimentation. The mystery to doing great investigate is to be able to switch from thought to result with the slightest conceivable delay.

Keras has the taking after primary characteristics:

- Allows the same code to run seamlessly on a CPU or a GPU.

- User-friendly API that creates it simple to rapidly test profound learning models.

from _ future__import print_function
import numpy as np
np.random.seed(1337) \# for reproducibility
from keras.datasets import mnist
from keras. models import Sequential
from keras. layers import Dense, Activation, Flatten
from keras.layers import Convolution2D,
MaxPooling2D
from keras.utils import np_utils
frnm kerac imnnrt harkand ac $K$

\subsection{TensorFlow:}

TensorFlow Serving could be a flexible, high-performance machine learning model serving system which is intended for production environments. TensorFlow Serving allows the deployment of recent algorithms and tests, while retaining the identical application architecture and APIs.

$$
\begin{aligned}
& \text { import tensorflow as tf } \\
& \text { from tensorflow. python.tools import freeze_graph } \\
& \text { from tensorflow. python.tools import optimize_for_inference_lib }
\end{aligned}
$$

\section{METHODS:}

In the Offline handwritten Hindi word recognition, we have used two approaches:

- K-Mean Clustering

- Support Vector Machine 


\subsection{K-Mean Clustering:}

K-Mean clustering is one of the foremost capable and common unsupervised machine learning calculations. The KMean calculations decides the $\mathrm{k}$ number of centroids, and in this way allots each information point to the closest cluster, hence keeping the centroids as little as possible.

For the handling of scholastic information, the K-Means calculations starts with an essential gather of arbitrarily chosen centroids, which are utilized as the beginning points for each cluster, and after that performs emphases(dreary) calculations to optimize the area of the centroids.

- Stops building and optimizing clusters when either:

- The number of emphases indicated has been achieved.

\section{Step1: Import Libraries:}

\begin{tabular}{l}
\hline import pandas as pd \\
import numpy as np \\
import matplotlib.pyplot as plt \\
from sklearn. cluster import KMeans \\
$\%$ matplotlib inline \\
\hline
\end{tabular}

We'll import the following libraries into our project, as you can see from the above code:

a) Pandas to read and write Tables

b) Numpy for effective computations

c) Matplotlib for data visualization.

\section{Step2: Generate aléatoire data:}

Here's the code for generating some random data in a space of two dimensions:

\section{Step3: Using Scikit-Learn:}

We will use some of the functions available in the Scikitlearn library to process the data generated at random.

\subsection{Support Vector Machine:}

Support vector machines (SVM) are a bunch of directed strategies of learning utilized for classification, relapse, and distinguishing proof of exceptions.

Support vector machines have the preferences of:

- Efficient in spaces with awesome measurements.

- Still fruitful in circumstances where the number of tests is more prominent than the number of estimations.

- Uses in choice work a sub-set of preparing focuses.

SVM can be of two kinds: Linear SVM and Non-Linear SVM

- Linear SVM: Linear SVM is utilized for directly distinct information, meaning that on the off chance that a dataset can be separated into two classes employing a single straight line, it is called straightly divisible information, and classifier is called a direct SVM classifier.

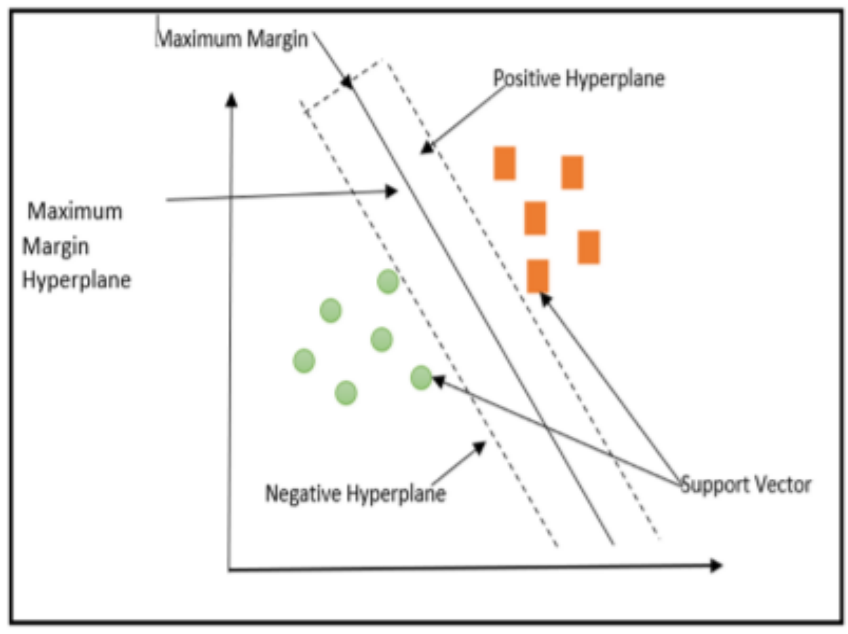

Figure. 4 Support Vector Machine

- Non-Linear SVM: Non-Linear SVM is utilized for non-linearly partitioned information, which implies that on the off chance that a dataset cannot be classified employing a straight line, that information is alluded to as non-linear information and the classifier utilized is alluded to as a non-linear classifier

Hyperplane: A few lines/decision boundaries can be utilized to recognize the bunches in n-dimensional space, but we got to discover the leading choice boundary to assist characterize the information focuses. This best restrain is known as SVM hyperplane.[4].

\subsection{Random Forest Classifier:}

Random forest could be a directional learning calculation both for classification and relapse. However, it is used mainly for classification problems. Be that as it may, it is utilized basically for classification issues. Since we know a forest comprises of trees and more trees implies more sound forest. It is an ensemble approach that's superior than a single choice tree, and by combining the result it diminishes the overfitting.

\section{Random Forest Algorithm Function}

Using the taking after steps, we are able get it the workings of the Random Forest algorithm:

Phase 1 - To begin with, choose the random tests from a given dataset.

Phase 2 Following, this calculation will make a choice tree for each test. At that point each choice tree will get the forecast result.

Phase 3 - Voting for any anticipated result will be done in this stage.

Phase 4 - Inevitably, choose the result of the foremost voted prediction as to the ultimate result of the prediction.

The following diagram shows its operation - 


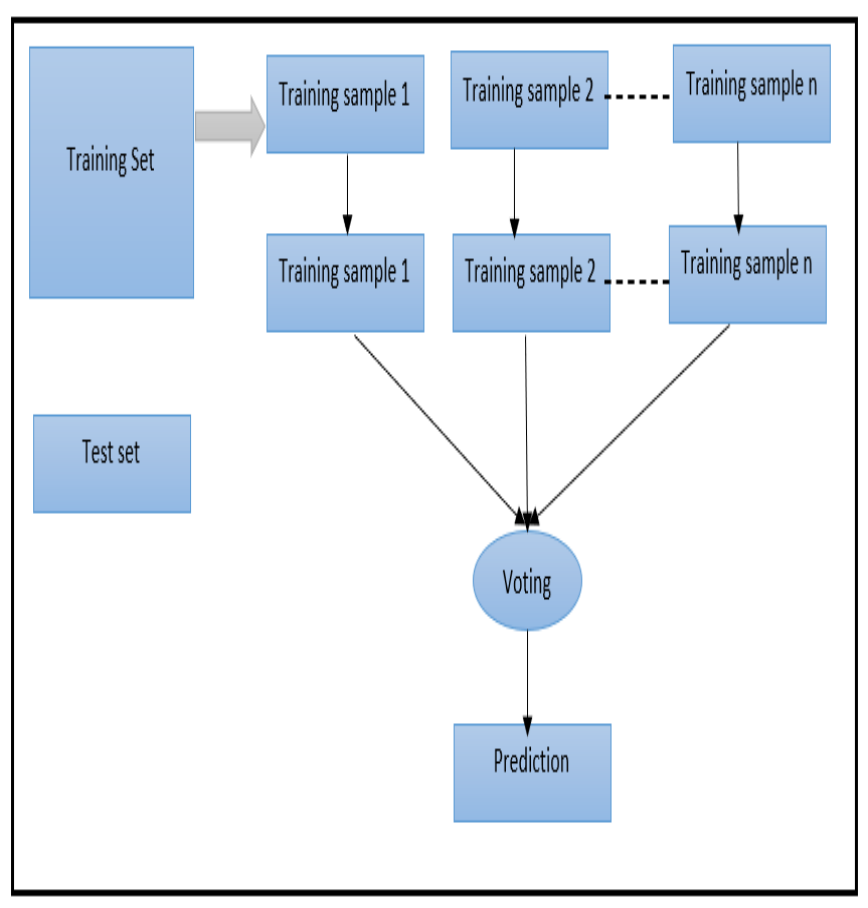

Figure. 5 Random Forest Classifier shows it operations

\begin{tabular}{|c|c|c|}
\hline Ref. No. & 1 & 2 \\
\hline $\begin{array}{l}\text { Technology } \\
\text { Used }\end{array}$ & K-Mean Clustering & $\begin{array}{ll}\text { Support } & \text { vector } \\
\text { machine }\end{array}$ \\
\hline Parameter & $\begin{array}{ll}- & \text { Pre-processing } \\
- & \text { Segmentation } \\
\text { - } & \text { Feature } \\
& \text { Extraction } \\
\text { - } & \text { Classification }\end{array}$ & $\begin{array}{l}\text { Handwritten character } \\
\text { recognition. } \\
\text { OCR } \\
\text { Feature extraction on } \\
\text { SVM }\end{array}$ \\
\hline Year & 2014 & 2012 \\
\hline Findings & $\begin{array}{l}\text { The address of Hindi } \\
\text { character acknowledgment } \\
\text { is tended to effectively with } \\
\text { the utilize of K implies. The } \\
\text { even and vertical angle for } \\
\text { the location of edges by } \\
\text { implies of a manual canny } \\
\text { edges finder and the vertical } \\
\text { and flat projection of each } \\
\text { character are utilized to } \\
\text { coordinate the other } \\
\text { characters and their } \\
\text { classifications. }\end{array}$ & $\begin{array}{l}\text { The most perfect way fo } \\
\text { the recognizable proof o } \\
\text { handwritten characters i. } \\
\text { the combination of the } \\
\text { SVM classifier and the } \\
\text { inclining include extractio }\end{array}$ \\
\hline
\end{tabular}

Table 1. K-Means Clustering and SVM [4]

\section{IMPLEMENTATION AND RESULT}

The python libraries Numpy, Panda, Matplotlib, Sckit-Learn, Keras () are utilized for tall execution and logical computation. The proposed work considered 166 diverse character classes, which comprises 32-character classes for Hindi. After resizing the pictures, the dataset was splitted into $70 \%$ pictures to be utilized for preparing and 30\% pictures forest and approval. As input picture shapes, pooling techniques, and optimizer capacities on the demonstrate. The learnable channels of $\mathrm{CNN}$ are analyzed such as input picture shapes, pooling procedures, and optimizer capacities on the show. The apperception exactness has identified when the demonstrate contains four convolutional layers, with zeropadding and max-pooling by two plenarily associated layers.

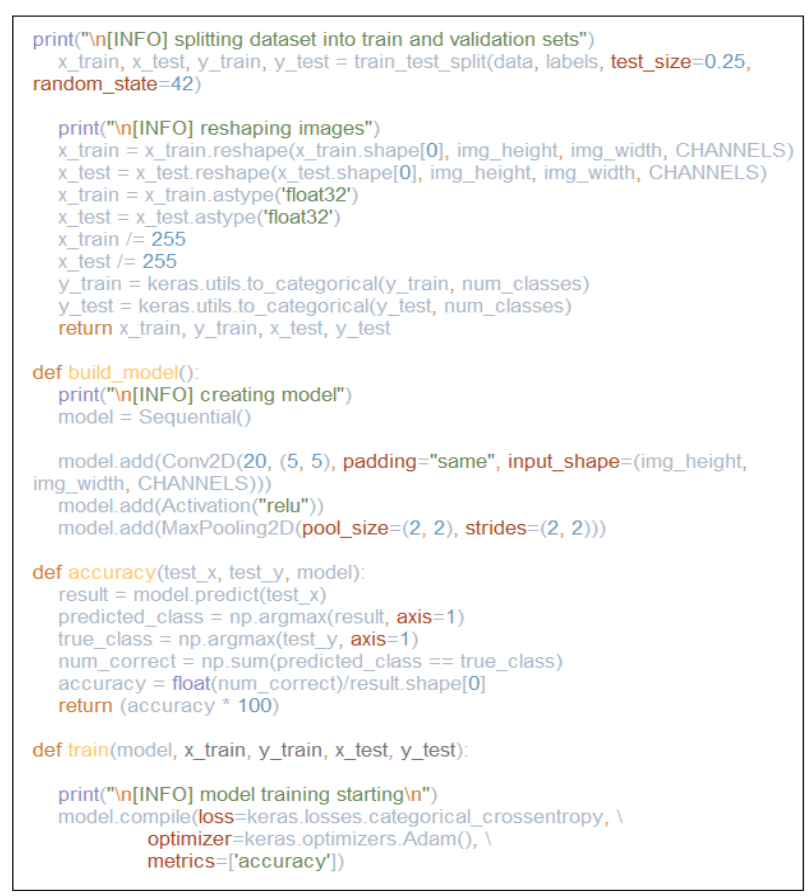

We have created the MNIST dataset that is as shown below:

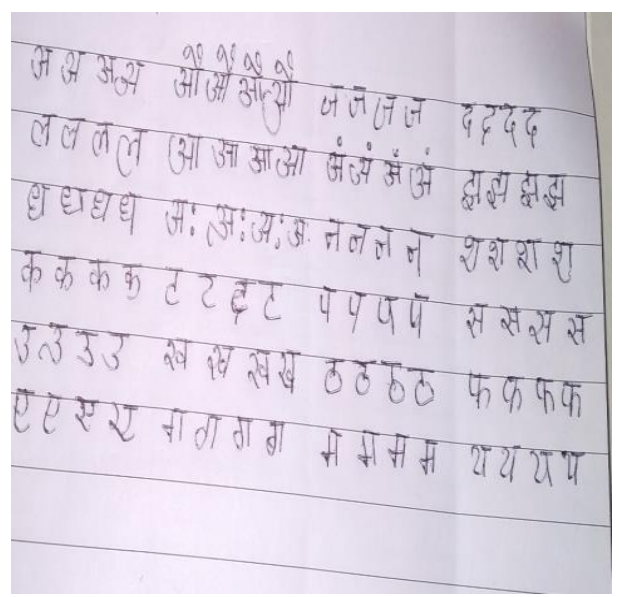

Figure 6. MNIST dataset

\begin{tabular}{|c|c|c|c|c|}
\hline $\begin{array}{c}\text { Expe } \\
\text { ri- } \\
\text { ment }\end{array}$ & Structure & Image & Classifier & Accuracy \\
\hline 1 & $\begin{array}{c}\text { CNN- Random } \\
\text { forest Classifier }\end{array}$ & 32 X 32 & $\begin{array}{c}\text { Random Forest } \\
\text { Classifier }\end{array}$ & $72.3 \%$ \\
\hline 2 & CNN-KNN & $32 \times 32$ & $\begin{array}{c}\text { K Neighbors } \\
\text { Classifier }\end{array}$ & $83.4 \%$ \\
\hline
\end{tabular}

Table 2. Accuracy by CNN for Hindi Classifier

\section{CONCLUSION}

Various papers have been distributed the region of optical character acknowledgement framework methods. We moreover learned from these articles that the different approaches too different ways of recognizing the character. In a organize, a printed report or a checked picture is changed to an ASCII character that can be recognized by a computer. Coordinating a format is the strategy of looking for a sub picture position that's a reference. The most perfect way to 
recognized handwritten characters is to combine SVM classifier with the inclining highlight extraction handle. Gee, neural systems, and their application for character acknowledgment. By segmentation prepare, able to isolate the touching characters and evacuate shrirorekh from the term. It is additionally accepted that k-means have a genuine degree of text style autonomy, which is aiming to diminish the scale of the preparing database.

\section{REFERENCES}

[1] Karishma Patel, Mikita Gandhi, "Offline Handwritten Character Recognition: A Review", Volume-7, May-2016.

[2] Sitaram Ramachandrula, Shrang Jain, Hariharan Ravishankar," Offline Handwritten Word Recognition in Hindi".

[3] P Sujatha, D. Lalitha Bhaskari, 'Telugu and Hindi Script Recognition through Deep Learning Techniques,' Volume-8 Issue11, September 2019.

[4] Sonika Dogra and Chandra Prakash, "PEHCHAAN: HINDI HANDWRITTEN CHARACTER RECOGNITION BASED ON SVM", Vol. 4 No. 05 May 2012.

[5] B. Baranidharan, Apoorva Kandpalb, Adhiraj Chakraborty," Hindi Handwritten Character Recognition Using Convolutional Neural Network". 